\title{
Increased number of $\mathrm{FoxP3}^{+} \mathrm{CD4}^{+}$regulatory $\mathrm{T}$ cells in inflammatory bowel disease
}

\author{
HIROMITSU BAN, AKIRA ANDOH, MAKOTO SHIOYA, ATSUSHI NISHIDA, \\ TOMOYUKI TSUJIKAWA and YOSHIHIDE FUJIYAMA
}

Department of Medicine, Shiga University of Medical Science, Seta Tukinowa, Otsu 520-2192, Japan

Received May 5, 2008; Accepted June 4, 2008

DOI: 10.3892/mmr_00000006

\begin{abstract}
FoxP3 is a member of the forkhead/winged helix family of transcription factors and plays a critical role in the development and function of $\mathrm{CD} 4{ }^{+} \mathrm{CD} 25^{+}$regulatory $\mathrm{T}$ cells (Tregs). In this study, we performed an immunohistochemical evaluation of FoxP3-expressing cells in inflammatory bowel disease (IBD) mucosa. Mucosal FoxP3 expression was evaluated by immunohistochemistry in samples from normal $(\mathrm{n}=30)$, ulcerative colitis (UC) $(\mathrm{n}=53)$ and Crohn's disease (CD) $(n=24)$ mucosa. There were no FoxP3-immunopositive cells in the normal colonic mucosa. In contrast, FoxP3immunopositive cells were significantly increased in the inflamed regions of patients with active UC and CD. FoxP3immunopositive cells completely coincided with some of the CD4-positive $\mathrm{T}$ cells. In conclusion, FoxP3-immunopositive Tregs were expanded in the inflamed mucosa of IBD patients. This suggests that these cells have impaired regulatory functions in the IBD mucosa.
\end{abstract}

\section{Introduction}

Inflammatory bowel disease (IBD), ulcerative colitis (UC) and Crohn's disease (CD) are chronic intestinal disorders of unknown etiology. The precise pathogenesis of IBD has not yet been elucidated, but the infiltration of leukocytes such as neutrophils, monocytes and lymphocytes into the intestinal mucosa plays an important role in the formation of mucosal lesions via the increased production of proinflammatory cytokines and inflammatory mediators $(1,2)$.

Three different $\mathrm{CD}^{+}{ }^{+}$regulatory $\mathrm{T}$ cell populations have been described as being capable of inhibiting $\mathrm{T}$ cell response $(3,4)$, i.e., inducible interleukin-10-producing $\mathrm{T}$ cells type 1 (Tr1), transforming growth factor- $\beta$-producing Th3 cells arising in the periphery, and naturally occurring thymus-derived

Correspondence to: Dr Akira Andoh, Department of Medicine, Shiga University of Medical Science, Seta Tukinowa, Otsu 520-2192, Japan

E-mail: andoh@belle.shiga-med.ac.jp

Key words: $\mathrm{CD}^{+}$regulatory $\mathrm{T}$ cells, inflammatory bowel disease regulatory $\mathrm{T}$ cells (Tregs) (5-10). The mechanisms by which Tregs mediate their suppressive effects are not fully understood. Their regulatory functions are mediated by the secretion of suppressive cytokines, as well as by cell contact-dependent signaling involving cytotoxic $\mathrm{T}$ lymphocyte-associated antigen 4, glucocorticoid-induced TNF receptor and, presumably, lymphocyte activation gene 3 (11-13).

FoxP3 is a member of the forkhead/winged helix family of transcription factors, and plays a critical role in the development and function of CD4 ${ }^{+} \mathrm{CD} 25^{+}$Tregs (14). CD4 ${ }^{+} \mathrm{CD} 25^{+}$ Tregs are mainly produced in the thymus, but can also be induced in the periphery under various stimulatory conditions. For example, in humans FoxP3 expression can be induced in $\mathrm{CD} 4^{+} \mathrm{CD} 25^{-} \mathrm{T}$ cells, whereby the cells acquire regulatory properties (15). The rationale for propagating gut-derived Tregs is that they have a high expression of gut-homing integrin $\alpha 4 \beta 7$, and may show a higher tendency to home to the inflamed gut mucosa than do Tregs from the peripheral blood with a skin-specific integrin expression (16). These gut-derived Tregs may be able to restore immune balance in IBD. A recent study has shown that the adoptive transfer of $\mathrm{CD} 4{ }^{+} \mathrm{CD} 25^{+}$Tregs can cure established experimental colitis, suggesting their potential utility as ex vivo-generated Tregs for the treatment of IBD (17).

Another recent study showed that, in UC patients, Tregs are increased in the lamina propria of the inflamed mucosa and in the mesenteric lymph nodes (18). However, changes in the number of Treg cells and their distribution in the intestinal mucosa of CD patients remain unknown. In this study, we performed an immunohistochemical study of FoxP3-expressing cells in the IBD mucosa.

\section{Materials and methods}

Antibodies and tissue samples. The following primary antibodies were used in this study: monoclonal mouse antihuman CD4 (Nichirei, Tokyo, Japan) and monoclonal rat-antihuman FoxP3 (eBioscience, San Diego, CA). The diagnosis for $\mathrm{UC}$ and $\mathrm{CD}$ was based on conventional clinical, endoscopic and histopathologic criteria. Surgically obtained specimens from 53 patients with UC and 24 patients with CD were used with informed consent. The ethics committee of the Shiga University of Medical Science approved this study. Normal colorectal tissues were obtained by surgical resection for colon cancer at distal tumor sites $(n=30)$. 

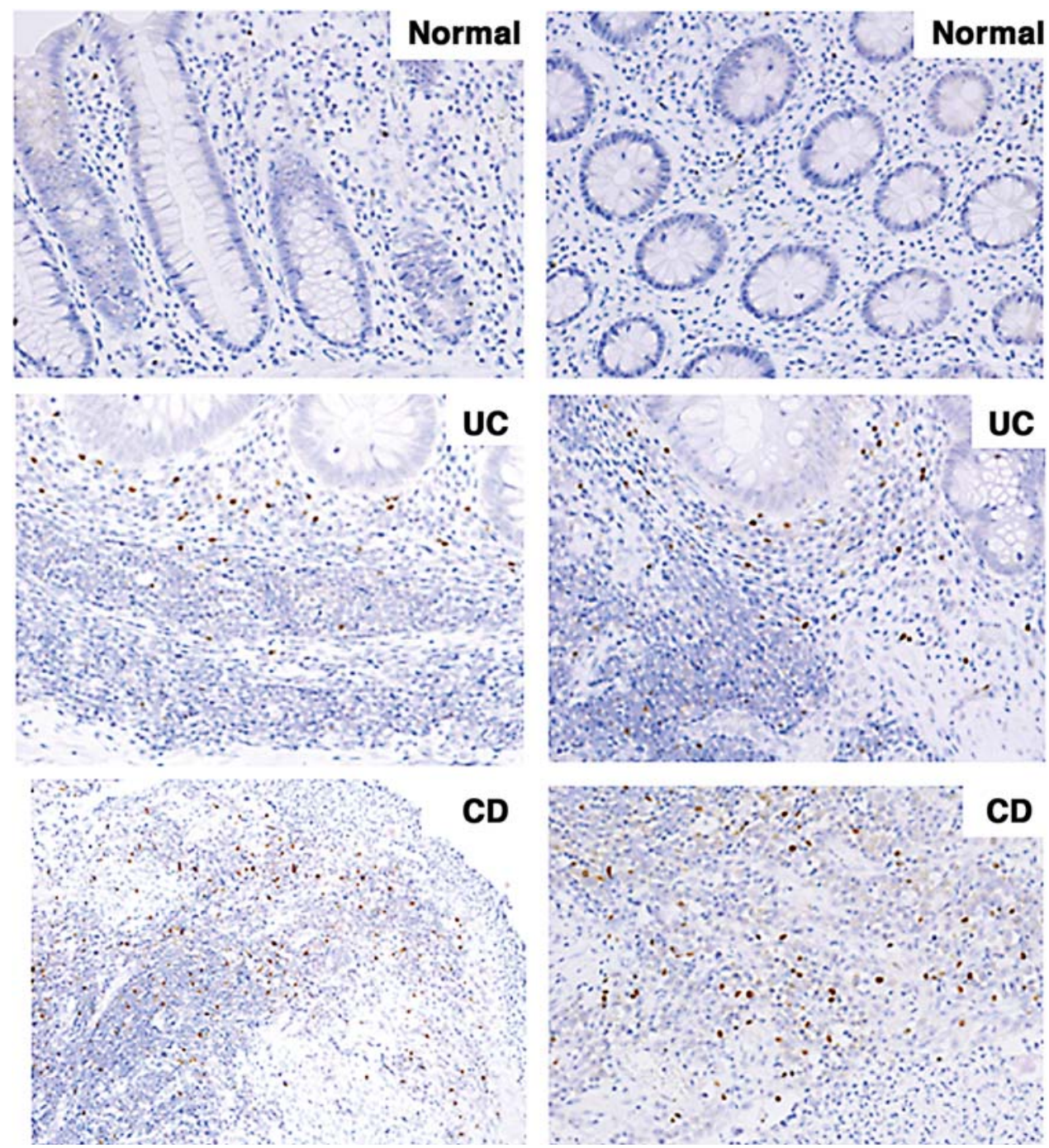

Figure 1. FoxP3-positive cells in normal colonic mucosa and in the inflamed mucosa of UC and CD patients (x200). UC, ulcerative colitis; CD, Crohn's disease
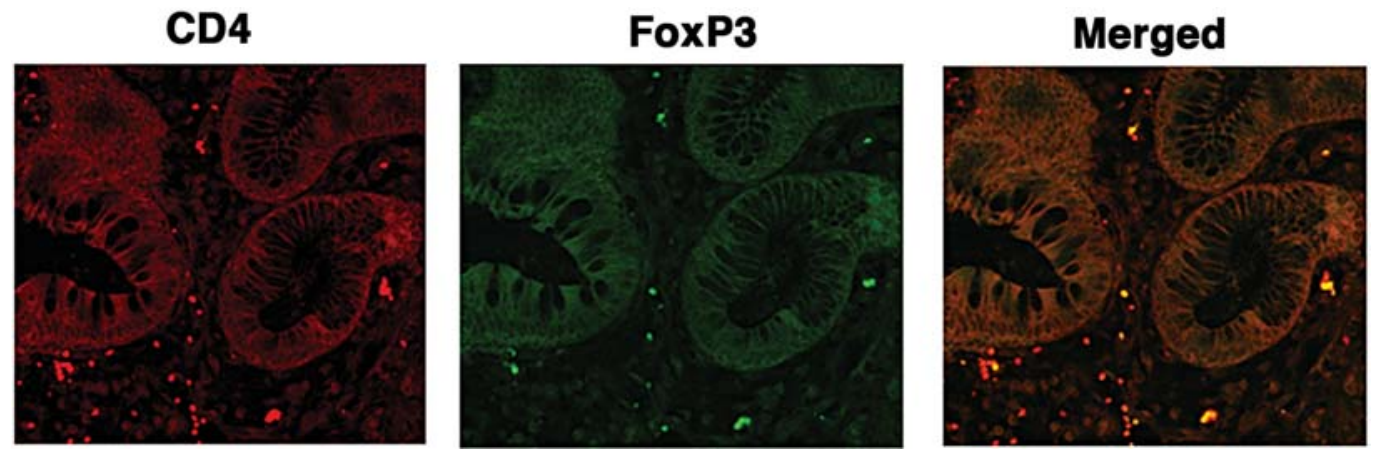

Figure 2. Double staining with anti-CD4 (red fluorescence) and FoxP3-positive cells (green fluorescence) in inflamed mucosa of a patient with Crohn's disease. CD4/FoxP3 double-positive cells were detected as yellow (far right).

Immunohistochemistry. After incubation with the primary antibodies, sections were treated with a biotin-conjugated goat anti-mouse IgG (Vector, Burlingame, CA) and avidin-biotinperoxidase complexes (ABC, Vector).

According to the method described by Middel et al (19), an evaluation of FoxP3 immunoreactivity was carried out by evaluators in a blinded manner. Corresponding areas of the tissue sections were marked, and high power fields were counted at $\times 400$ magnification. The mean count from a total of five high power fields per slide was used.

For double staining procedures, FITC-labeled anti-human FoxP3 antibodies were applied and incubated overnight at $4^{\circ} \mathrm{C}$ in a humidified chamber. Subsequently, monoclonal mouse anti-human CD4 antibodies (Nichirei) were applied and incubated overnight at $4^{\circ} \mathrm{C}$. Cy3-labeled anti-mouse $\mathrm{IgG}$ antibodies were applied for $60 \mathrm{~min}$ at room temperature. The 


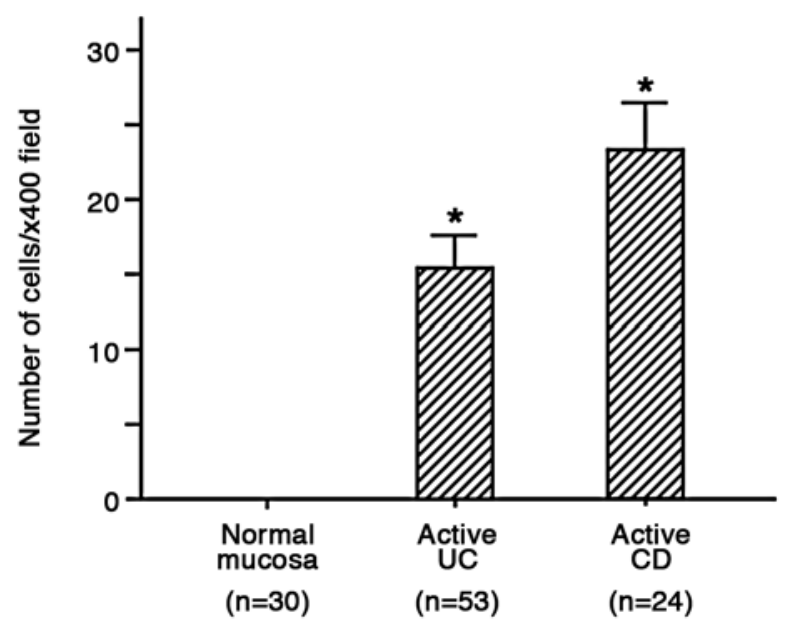

Figure 3. The number of FoxP3-positive cells in normal, active ulcerative colitis (UC) and active Crohn's disease (CD) mucosa. Results were expressed as the number of positive cells per field $(x 400)$. Data were expressed as the mean $\pm \mathrm{SD}$.

images were captured using the digital confocal laser scanning system MRC-600 (Bio Rad, Hercules, CA).

Statistical analysis. The statistical significance of the differences was determined by the Mann-Whitney U test (Statview Version 4.5). Differences resulting in P-values $<0.05$ were considered to be statistically significant.

\section{Results}

To evaluate FoxP3-immunopositive cells in the mucosa, the samples were immunostained with anti-FoxP3 antibodies. As shown in Fig. 1, there were no FoxP3-immunopositive cells in the normal colonic mucosa. In contrast, FoxP3-immunopositive cells were increased in the inflamed regions of patients with active UC and CD (Fig. 1).

To confirm the cellular origin of the FoxP3-immunopositive cells in the IBD mucosa, samples from patients with active CD were double-immunostained with fluorescencelabeled anti-CD4 (specific for T cells) and anti-human FoxP3 antibodies (Fig. 2). There were numerous CD4-immunopositive T cells (red fluorescence; Fig. 2) and FoxP3-immunopositive cells (green fluorescence; Fig. 2). CD4/FoxP3 doubleimmunopositive cells were detected as yellow, and the FoxP3immunopositive cells completely coincided with some of the CD4-positive T cells.

As shown in Fig. 3, the number of FoxP3-immunopositive cells increased significantly in the active lesions from IBD patients.

\section{Discussion}

Tregs control immune responses to self- and foreign antigens, and are likely candidates in the search for inadequate counter regulation in IBD. They are also involved in oral tolerance (20), the inhibition of Th1 cell activation against enteric bacterial antigens (21), and the prevention of colitis in animal models of IBD (22). Recent reports have demonstrated that the transcription factor FoxP3 is a specific marker for Tregs (14). In contrast to most phenotypic markers such as CD25, the expression of FoxP3 seems to be crucial to their functional characteristics. This was demonstrated by the introduction of the FoxP3 gene into naive $\mathrm{CD} 4{ }^{+} \mathrm{CD} 25^{+} \mathrm{T}$ cells, which subsequently displayed suppressive functions $(14,23)$.

In this study, we showed that FoxP3-immunopositive cells increased in number in the inflamed mucosa of IBD patients. Previously, Yu et al reported an increased number of FoxP3immunopositive cells in the mucosa and mesenteric lymph nodes of CD patients (18). Our observations are compatible with their findings, and extend them to similar phenomena in UC patients. Previous studies have demonstrated that Tregs inhibit the stimulatory functions of dendritic cells, accounting for their major regulatory functions in the differentiation and proliferation of effector T cells (14). An increased number of FoxP3-immunopositive cells in the lamina propria of IBD patients suggests an interaction between these cells and antigen-presenting dendritic cells and, consequently, a suppression of immune responses in the mucosa. However, the reasons for the failure of FoxP3-immunopositive cells to suppress the recurrence of colitis remain unclear. Investigators suggest that the suppressive activity of FoxP-immunopositive cells is down-regulated by other signals, such as toll-like receptors $(24,25)$. This suggests the possibility of a functional down-regulation of FoxP3-immunopositive cells in the IBD mucosa.

In conclusion, FoxP3-immunopositive Tregs were expanded in the inflamed mucosa of IBD patients. Functional analysis of FoxP3-immunopositive cells isolated from the IBD mucosa may be helpful in clarifying the significance of the increased number of cells in IBD mucosa.

\section{References}

1. Mizoguchi A and Mizoguchi E: Inflammatory bowel disease, past, present and future: lessons from animal models. J Gastroenterol 43: 1-17, 2008.

2. Sands BE: Inflammatory bowel disease: past, present, and future. J Gastroenterol 42: 16-25, 2007.

3. Jonuleit $\mathrm{H}$ and Schmitt E: The regulatory $\mathrm{T}$ cell family: distinct subsets and their interrelations. J Immunol 171: 6323-6327, 2003.

4. Lio CW and Hsieh CS: A two-step process for thymic regulatory T cell development. Immunity 28: 100-111, 2008.

5. Carrier Y, Yuan J, Kuchroo VK and Weiner HL: Th3 cells in peripheral tolerance. I. Induction of Foxp3-positive regulatory T cells by Th3 cells derived from TGF-beta T cell-transgenic mice. J Immunol 178: 179-185, 2007.

6. Carrier Y, Yuan J, Kuchroo VK and Weiner HL: Th3 cells in peripheral tolerance. II. TGF-beta-transgenic Th3 cells rescue IL-2-deficient mice from autoimmunity. J Immunol 178: 172-178, 2007.

7. Awasthi A, Carrier Y, Peron JP, et al: A dominant function for interleukin 27 in generating interleukin 10-producing antiinflammatory T cells. Nat Immunol 8: 1380-1389, 2007.

8. Wu K, Bi Y, Sun K and Wang C: IL-10-producing type 1 regulatory T cells and allergy. Cell Mol Immunol 4: 269-275, 2007.

9. Burchill MA, Yang J, Vang KB, et al: Linked T cell receptor and cytokine signaling govern the development of the regulatory T cell repertoire. Immunity 28: 112-121, 2008.

10. Pallandre JR, Brillard E, Crehange G, et al: Role of STAT3 in $\mathrm{CD} 4{ }^{+} \mathrm{CD} 25^{+} \mathrm{FOXP}^{+}$regulatory lymphocyte generation: implications in graft-versus-host disease and antitumor immunity. $\mathbf{J}$ Immunol 179: 7593-7604, 2007.

11. Izcue A and Powrie F: Special regulatory T-cell review: regulatory $\mathrm{T}$ cells and the intestinal tract - patrolling the frontier. Immunology 123: 6-10, 2008

12. Read S, Malmstrom V and Powrie F: Cytotoxic T lymphocyteassociated antigen 4 plays an essential role in the function of $\mathrm{CD} 25(+) \mathrm{CD} 4(+)$ regulatory cells that control intestinal inflammation. J Exp Med 192: 295-302, 2000. 
13. McHugh RS, Whitters MJ, Piccirillo CA, et al: CD4(+)CD25(+) immunoregulatory $\mathrm{T}$ cells: gene expression analysis reveals a functional role for the glucocorticoid-induced TNF receptor. Immunity 16: 311-323, 2002.

14. Hori S, Nomura T and Sakaguchi S: Control of regulatory T cell development by the transcription factor Foxp3. Science 299: 1057-1061, 2003.

15. Walker MR, Kasprowicz DJ, Gersuk VH, et al: Induction of FoxP3 and acquisition of $\mathrm{T}$ regulatory activity by stimulated human CD4+CD25- T cells. J Clin Invest 112: 1437-1443, 2003.

16. Iellem A, Colantonio L and D'Ambrosio D: Skin-versus gutskewed homing receptor expression and intrinsic CCR4 expression on human peripheral blood $\mathrm{CD} 4{ }^{+} \mathrm{CD} 25^{+}$suppressor $\mathrm{T}$ cells. Eur J Immunol 33: 1488-1496, 2003.

17. Fantini MC, Becker C, Tubbe I, et al: Transforming growth factor beta induced FoxP3 ${ }^{+}$regulatory $\mathrm{T}$ cells suppress Th1 mediated experimental colitis. Gut 55: 671-680, 2006.

18. Yu QT, Saruta M, Avanesyan A, Fleshner PR, Banham AH and Papadakis KA: Expression and functional characterization of $\mathrm{FOXP}^{+} \mathrm{CD}^{+}{ }^{+}$regulatory $\mathrm{T}$ cells in ulcerative colitis. Inflamm Bowel Dis 13: 191-199, 2007.
19. Middel P, Reich K, Polzien F, et al: Interleukin 16 expression and phenotype of interleukin 16 producing cells in Crohn's disease. Gut 49: 795-803, 2001.

20. Zhang X, Izikson L, Liu L and Weiner HL: Activation of $\mathrm{CD} 25(+) \mathrm{CD} 4(+)$ regulatory $\mathrm{T}$ cells by oral antigen administration. J Immunol 167: 4245-4253, 2001.

21. Gad M, Brimnes J and Claesson $\mathrm{MH}$ : $\mathrm{CD}^{+}{ }^{+} \mathrm{T}$ regulatory cells from the colonic lamina propria of normal mice inhibit proliferation of enterobacteria-reactive, disease-inducing Th1-cells from scid mice with colitis. Clin Exp Immunol 131: 34-40, 2003.

22. Mottet C, Uhlig HH and Powrie F: Cutting edge: cure of colitis by $\mathrm{CD} 4{ }^{+} \mathrm{CD} 25^{+}$regulatory T cells. J Immunol 170: 3939-3943, 2003.

23. Yagi H, Nomura T, Nakamura K, et al: Crucial role of FOXP3 in the development and function of human $\mathrm{CD} 25^{+} \mathrm{CD} 4^{+}$regulatory T cells. Int Immunol 16: 1643-1656, 2004.

24. Pasare $C$ and Medzhitov R: Toll pathway-dependent blockade of $\mathrm{CD}^{+} \mathrm{CD} 25^{+} \mathrm{T}$ cell-mediated suppression by dendritic cells. Science 299: 1033-1036, 2003.

25. Sutmuller RP, den Brok MH, Kramer M, et al: Toll-like receptor 2 controls expansion and function of regulatory $\mathrm{T}$ cells. J Clin Invest 116: 485-494, 2006. 